\title{
Towards Virtual Testing of Textile Composites: Calibration of Thermoelastic
} Tow Properties

\author{
John H. Shaw, Varun P. Rajan, Matthew Blacklock, and Frank W. Zok ${ }^{\dagger}$ \\ Materials Department, University of California, Santa Barbara, California 93106
}

\begin{abstract}
We present a methodology for calibrating the thermoelastic properties of constituent tows in a woven $\mathrm{C} / \mathrm{SiC}$ composite using a combination of experimental measurements and finite element simulations of strain distributions that arise upon heating. Because of the nonuniform distribution of the matrix phase and the presence of matrix microcracks, tow properties cannot be reliably predicted a priori using micromechanical models alone; instead, some can only be inferred from coupled experimental/numerical studies of the kind presented here. A small number of iterations of finite element simulations is required to achieve satisfactory agreement in all thermal strain components. The number of iterations is minimized by first performing simulations using model-based estimates of the effective tow properties, followed by judicious adjustments to select property values. Comparisons between measurements and simulations are performed on the basis of strains averaged over a characteristic area dictated by tow dimensions. The study reveals that, although each segment of a particular tow genus is nominally equivalent to all others, the strains within each population exhibit considerable variation. This is attributable to variations in both tow geometry and connectivity between adjacent tows. The virtual tests predict strain variations of comparable magnitude.
\end{abstract}

\section{Introduction}

$\mathrm{O}$ NE of the current challenges in the ceramics community is the development of a capability for performing highfidelity virtual tests on fiber-reinforced ceramic-matrix composites (CMCs). The potential payoffs of such a capability are enormous. First, in light of the high cost of the constituents and the specialized facilities required for processing these materials, real tests at the scale and in the number historically used to qualify metallic alloys are prohibitive. Replacing some of these with virtual tests could significantly reduce the high costs of material qualification. Indeed, one role of virtual tests is to inform and guide a real test plan so that material is utilized most efficiently. Second, a virtual test can probe local stresses and strains throughout the entire material under service conditions and can thereby identify likely locations and modes of material failure. In real tests, there are severe restrictions on the extent to which the interior of materials can be probed, especially at high temperatures. A virtual test can also probe effects of material defects and heterogeneities - features replete in ceramic compositeson thermal and mechanical performance. Such effects are rarely amenable to systematic experimental investigation. Finally, virtual tests can most efficiently identify optimal weaves of woven CMCs to match anticipated service loads.

E. Lara-Curzio - contributing editor

Manuscript No. 33459. Received July 2, 2013; approved December 29, 2013.

†Author to correspondence should be addressed. e-mail: zok@engineering.ucsb.edu
As part of a broad effort towards this challenge among several research groups in the National Hypersonics Science Center, the present study is focused on advancing one specific facet of the virtual test: that of the thermal expansion behavior of a highly anisotropic $\mathrm{C} / \mathrm{SiC}$ composite. This composite exhibits complexity over a range of length scales and with respect to several physical and mechanical phenomena. First, as with all textile composites, internal stresses resulting from a uniform macroscopic thermal or mechanical load are inherently nonuniform and depend on the weave architecture. Second, the constituents exhibit large mismatch in their respective coefficients of thermal expansion (CTE); typically, the axial and radial CTE of the fibers are about zero and $9 \times 10^{-6} \mathrm{~K}^{-1}$, respectively, whereas that of $\mathrm{SiC}$ is about $4 \times 10^{-6} \mathrm{~K}^{-1}$. Thus, for a thermal excursion of $1500 \mathrm{~K}$, the axial misfit strain between the constituents is about 0.006: likely sufficient to initiate local failure. Third, because of the nature of the methods employed in CMC processing, the matrix material tends to be distributed nonuniformly. For instance, during the chemical vapor infiltration (CVI) process commonly used in forming a $\mathrm{SiC}$ matrix, the tows can become pinched off from their surroundings once a sufficiently thick shell of $\mathrm{SiC}$ has formed on the tow surface, thereby preventing further ingress of the gases needed for densification. This has important implications regarding the anisotropy of the CTE and the stiffness of the tows as well as the anisotropy of thermal conductivity. Virtual tests are therefore highly desirable for probing the many facets of thermomechanical response of these composites.

The specific goal of the present study is to assess the capability of a virtual test in predicting the tow-scale thermal strains in a partially densified $\mathrm{C} / \mathrm{SiC}$ composite. The study builds on previous work in which the weave geometry of the same composite was characterized in detail by X-ray computed tomography $(\mathrm{CT})^{1}$ and parallel efforts to develop a virtual geometry generator. ${ }^{2-4}$ It also builds on recent advancements in testing capabilities: notably, full-field strain mapping at sub-tow length scales, at temperatures up to $1500^{\circ} \mathrm{C}$.

The article is organized in the following way. Section II describes the material of interest, the testing and analysis protocols, and the experimental measurements. Section III focuses on the virtual tests. Here, the goal is to identify the thermoelastic constants of the fiber tows that yield predicted fields that are consistent with the experimental measurements. One practical challenge is that the calibration is essentially underconstrained: the number of unknown variables exceeding the number of discriminating tests.

\section{Experimental Investigations}

\section{(1) Material}

The material examined in this study is a woven $\mathrm{C}$-fiber $\mathrm{SiC}$ matrix composite. ${ }^{1-3}$ Several views of the composite and some of the salient microstructural features are shown in Figs. 1 and 2. The fiber preform consists of T300-6K tows in a three-layer angle interlock weave. The tows fall into one of 


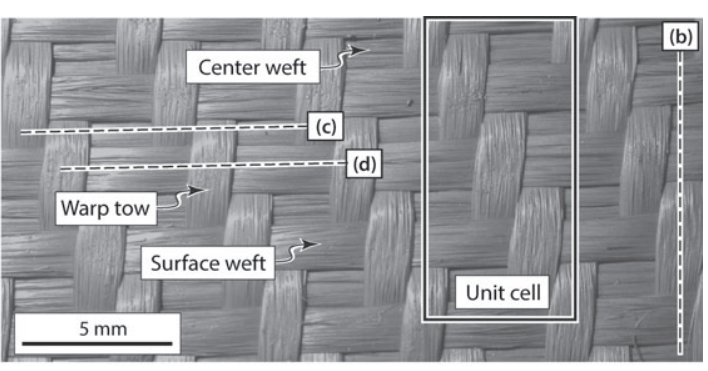

(a)

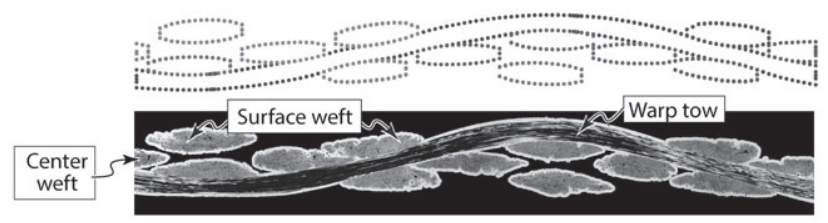

(b)

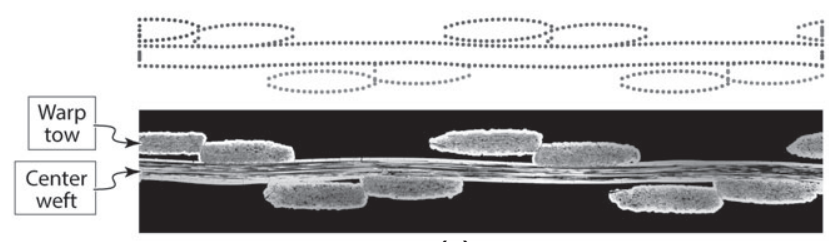

(c)

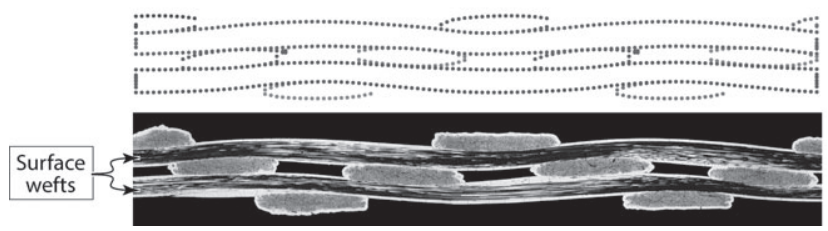

(d)

Fig. 1. (a) Plan view of the woven $\mathrm{C} / \mathrm{SiC}$ composite, indicating the various tow genuses and the unit cell. The lines denote the locations at which subsequent sections (b-d) were obtained. Also shown in (b-d) are corresponding arrays of surface nodes defining the tow boundaries used in the virtual tests.

three nominally equivalent types: (i) surface wefts, one set evident on each of the two surfaces, (ii) central wefts, nominally straight and residing midway between the two surface weft populations, and (iii) warp tows that weave through-thickness, transverse to the wefts. The fibers had been coated with a thin layer of pyrolytic carbon via CVI. The preform was then partially infiltrated with CVI SiC, resulting in thin nonuniform coatings on the individual fibers within the fiber tows and a relatively uniform 30-40 $\mu \mathrm{m}$-thick matrix layer around the fiber tows. The volume fraction of fibers, taken as the ratio of area occupied by fibers to the area contained within (but excluding) the $\mathrm{SiC}$ jacket, was $61 \%$; neglecting the very small amount of $\mathrm{SiC}$ within the tows, the inter-tow porosity was therefore taken to be $39 \%$. In this (partially -processed) state - that is, lacking a fully dense matrix - the effects of the anisotropic thermoelastic properties of the tows are manifested strongly in the spatial distributions of the thermal strains measured on the external surfaces (the presence of a dense matrix between tows would mask such effects).

\section{(2) Heating and Thermal Strain Measurements}

Thermal strains were measured using a laser heating and imaging system, shown in Fig. 3 and described elsewhere. ${ }^{5}$ Briefly, heating was accomplished with a $2 \mathrm{~kW} \mathrm{CO}$ laser operating at a wavelength of $10.6 \mu \mathrm{m}$. Custom optics were utilized to homogenize the beam. Temperature measurements were made using a combination of a two-color pyrometer operating at 800 and $950 \mathrm{~nm}$, a two-color spot pyrometer operating at $800 \mathrm{~nm}$ and $1.7 \mu \mathrm{m}$, and a single-color infrared camera operating at $3-5 \mu \mathrm{m}$.

Illumination for optical imaging and strain mapping was accomplished using an array of blue LEDs operating in the wavelength range $440-460 \mathrm{~nm}$. Imaging was performed with a pair of optical cameras fitted with $100 \mathrm{~mm}$ macrolenses. Band-pass filters were attached to the lenses to limit the wavelength of reflected and emitted light to a narrow band bracketing the LED output. Effects of thermal turbulence were minimized using an Ar gas knife blowing tangentially to the sample surface. The Ar also mitigates to some extent the rate of sample oxidation. In the present experiments, the magnification of the optical imaging system was $11 \mu \mathrm{m} /$ pixel at a standoff distance of $32 \mathrm{~cm}$. Following the experiment, the sequence of images was correlated using the VIC-3D DIC software (Correlated Solutions Inc., Columbia, SC).

Test samples $12 \mathrm{~mm} \times 12 \mathrm{~mm}$ in size were machined from the composite plate. The samples were speckled using an alumina slurry (Pyro-Paint 634-AL; Aremco Products Inc., Valley Cottage, NY) sprayed through an airbrush. The average speckle size, measured using an autocorrelation technique, was $100 \mu \mathrm{m}$. The samples were placed in a $12.7 \mathrm{~mm} \times$ $12.7 \mathrm{~mm} \times 2 \mathrm{~mm}$ deep well cut into a sheet of graphite. The well dimensions were selected to be slightly greater than the sample size to prevent intimate contact between the sample edges and the graphite (and hence minimize heat conduction) as well as allow thermal expansion to occur without constraint. The graphite sheet was backed by a plate of low-conductivity porous $\mathrm{SiC}$.

Samples were heated at a rate of $300 \mathrm{~K} / \mathrm{min}$ up to a target maximum temperature of $1000^{\circ} \mathrm{C}$, held at fixed heat flux for
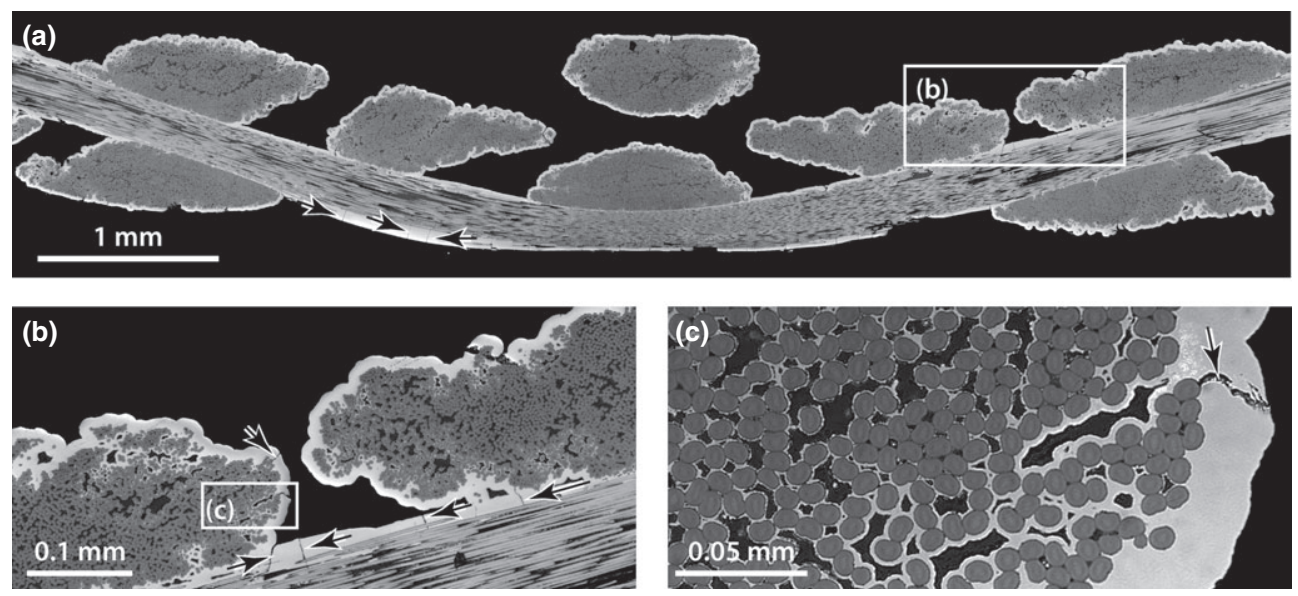

Fig. 2. Closer views of the tows, showing intra-tow porosity, accumulation of $\mathrm{SiC}$ on individual fibers within the interior of the tows and as a "jacket" on the tow exterior, and cracks in the SiC (indicated by arrows). 


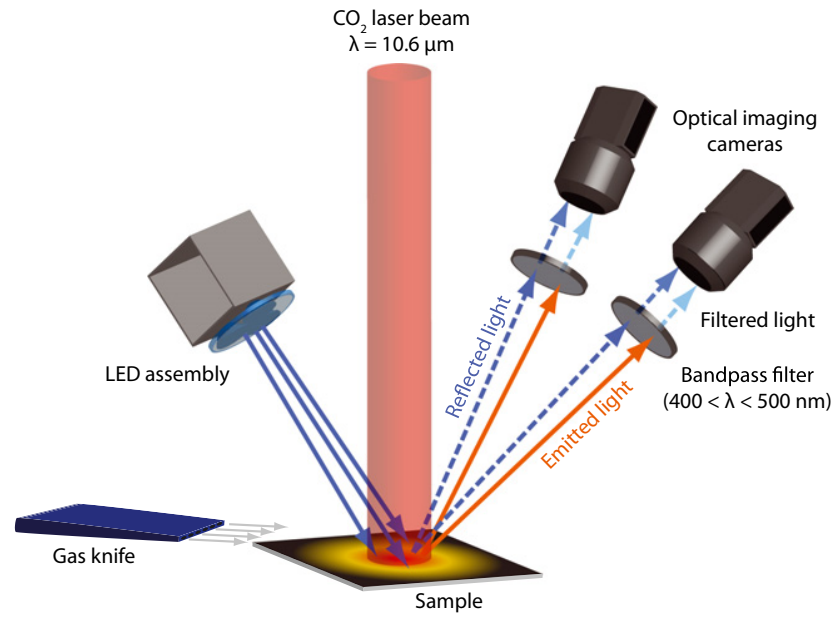

Fig. 3. Schematic of test apparatus used to map thermal strains.

3 min (to reach thermal equilibrium), and then allowed to cool. The strains were measured after thermal equilibrium had been attained. The average temperature measured on three such samples was $1026 \pm 10^{\circ} \mathrm{C}$. The temperature profile on the sample surface was monitored using the IR camera and was verified to be uniform within $\pm 10 \mathrm{~K}$. In combination with a thermocouple placed between the specimen and the back plate, the thermal images showed that the throughthickness temperature difference was no greater than $90 \mathrm{~K}$. Weight measurements before and after testing revealed only a small mass loss $(4 \%-7 \%)$. Inspection of the samples after testing indicated that the loss was due mainly to oxidation of the $\mathrm{C}$ fibers that had been exposed at the cut edges. Correlations of images taken at ambient and peak temperatures were performed using a subset size of 41 pixels $(0.47 \mathrm{~mm})$ and a step size of 4 pixels.

\section{(3) Analysis Procedures}

Following correlation, the displacement data were imported into Mathematica ${ }^{\circledR}$ (Wolfram Research, Champaign, IL) for segmentation and strain analysis. For quantitative comparisons with the virtual tests (described below), the DIC results were used to compute separately the average in-plane thermal strains within individual segments of both warp and surface weft tows on the sample surface (although narrow segments of central wefts were also visible, their area proved to be insufficient to make reliable strain measurements). To facilitate the latter computations, the DIC data were segmented using the following procedures.

First, weft tows were identified manually by inspecting surface height maps. Each segment was assigned a rectangular domain defined by the tow edges. Then, to eliminate the effects of neighboring tows on the displacement data within any one tow segment, each region of interest was pared by an amount equal to half the subset size around its periphery. In this way, none of the nodal data are affected by points residing in neighboring segments. Figure 4 shows an example of the resulting segmented wefts. An alternative technique was employed for segmenting the warp tows. With the warp crowns lying above the other tow segments, the warp segments were isolated from all others by thresholding the surface height $(z)$ data accordingly. A clustering algorithm was then employed to identify the data points that belong to each warp tow segment. The $z$-threshold was selected to remove regions lying within half the subset size of the tow edges. Finally, to remove segments that intersect the sample edges, segments with areas less than $80 \%$ of their respective genus average were excluded from further analysis. The results of the latter segmentation are also shown in Fig. 4.

The average in-plane strains in each tow segment were determined by first performing a linear fit to the displacement-position data, $u(x, y)$ and $v(x, y)$. The average in-plane strains, $\varepsilon_{x x}, \varepsilon_{y y}$, and $\varepsilon_{x y}$, were computed from displacement gradients in the usual way. The two normal strains (expressed in terms of the global $x-y$ coordinate system) were then classified according to their directions with respect to the local fiber direction: axial, if parallel to the tow trajectory and transverse, if perpendicular to it. Thus, in total, six populations of strain measurements were obtained: three each (axial, transverse, and shear) for the warp and the weft tows. Data for three test specimens were pooled together, yielding about 20 individual measurements for each distribution.

Comparisons of sequential image pairs at peak temperature indicated a root mean squared (RMS) displacement error $\left(v_{\text {RMS }}\right)$ of $0.01-0.02$ pixels, consistent with previous estimates. ${ }^{6}$ The resulting strain error scales with $v_{R M S} / h_{f}$, where $h_{f}$ is a characteristic dimension used for strain averaging (i.e., the gauge length). ${ }^{6,7}$ For gauge lengths employed in computing the average tow-segment strains, the strain error is estimated to be in the range of $(1-3) \times 10^{-4}$.

\section{(4) Experimental Results}

Maps of normal strains and surface height for a representative test sample are shown in Fig. 5. Superimposed on the maps are the approximate locations of the boundaries of the surface wefts and the warp tows. Upon inspection, it is apparent that the tows exhibit anisotropic thermal expansion. For instance, the strain $\varepsilon_{y y}$ in the weft tows is about twice that in the warp tows [left image of Fig. 5(b)]. Conversely, the strain $\varepsilon_{x x}$ in the weft tows is about half that in the warp tows. Indeed, these patterns coupled with the fine-scale intratow variations served as the impetus to compute

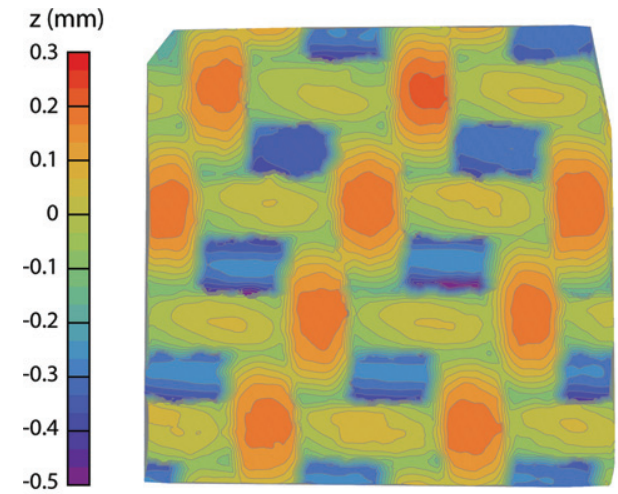

(a) Original topographic map

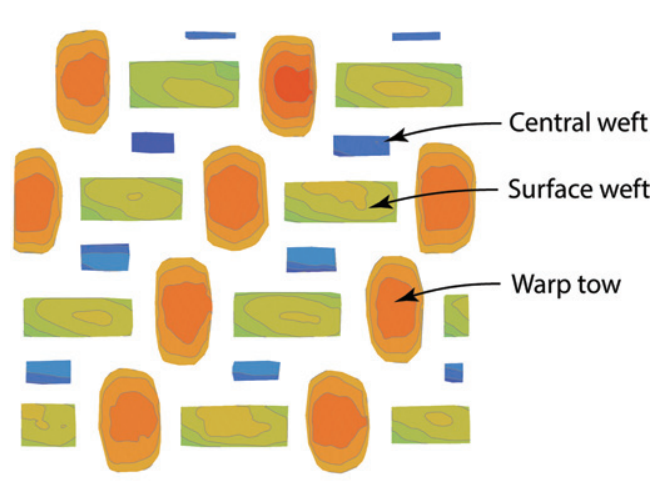

(b) After segmentation

Fig. 4. Topographic map of (a) an entire test coupon and (b) the same coupon after segmentation and segment paring. 


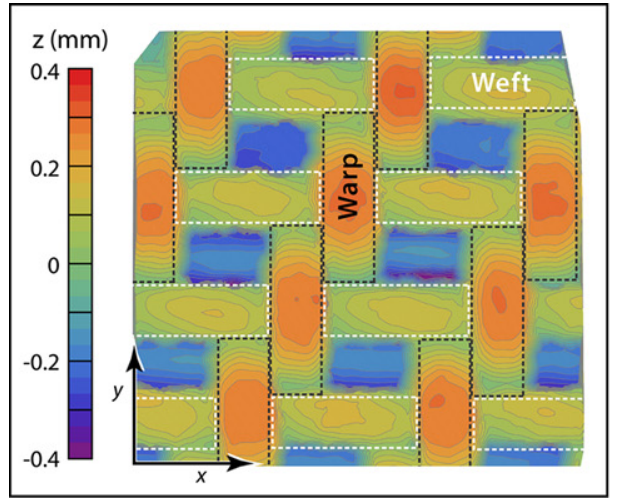

(a)

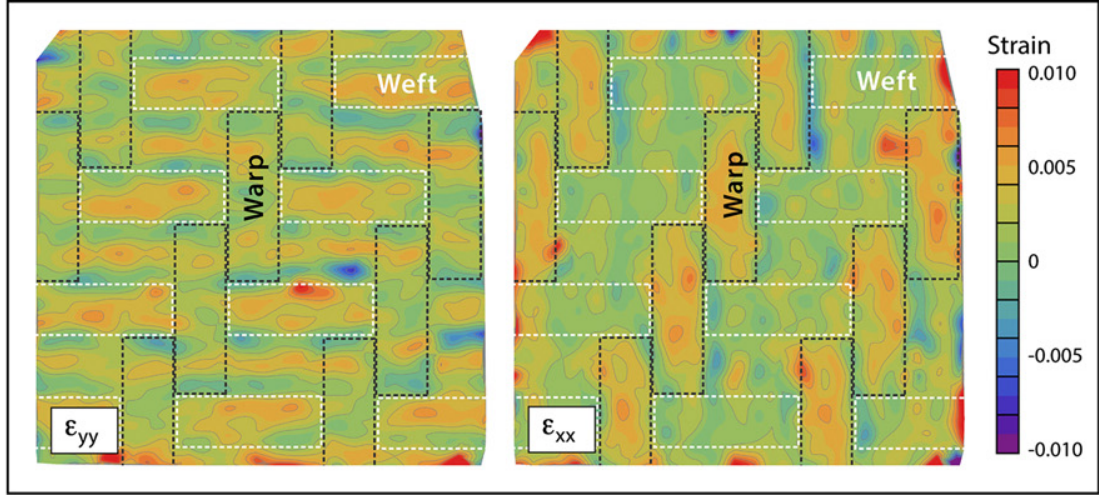

(b)

Fig. 5. (a) Topographic and (b) thermal strain maps of a representative test sample after heating from ambient temperature to $1026^{\circ} \mathrm{C}$.

area-averaged strains within individual tow segments; the latter were then used in comparisons with virtual test results.

The strain values obtained by fitting the displacement fields over individual tow segments are summarized in Fig. 6. They are presented in two forms. In (a) and (b), axial and transverse normal strains in both warp and surface weft tows are plotted against the distance from the sample center to the point of interest. The objective was to assess whether some of the variation was due to edge effects and, if so, the spatial extent to which the edge effects persist into the sample. Within the scatter in the measurements, no such effects are observed. In (c), normal and shear strains are represented by box-and-whisker plots. Here, each shaded box represents the middle 50 percentile of the distribution, the central line is the median, and the whiskers extending from the box encompass the first and fourth quartiles. The average axial and transverse strains for both tow types are about 0.002 and 0.0045 , respectively. The variations over the full range fall within about \pm 0.001 of the respective median. These variations are larger than those expected from measurement error alone (the latter would be of the order of twice the estimated error in strain measurement: $0.0002-0.0006)$. The inference is that the measured strain variations are likely intrinsic to the composite and not solely a manifestation of measurement error.

\section{Virtual Tests}

\section{(1) Specimen and Mesh Generation}

A finite element (FE) mesh of the angle interlock composite for use in the virtual tests was created using the virtual specimen generator. ${ }^{4}$ The first stage in a series of computational algorithms is the gathering of a statistical description of the tow architecture through analysis of X-ray CT images. ${ }^{1}$ The resulting positions and cross sections of each genus of tow (warp, central weft, top surface weft, and bottom surface weft) throughout the material are described by both a deterministic systematic field and nondeterministic stochastic variations. Tows are described in terms of six variables: the center of mass in space $\{x, y, z\}$, the cross-sectional area and aspect ratio, and the rotation of the major axis of the tow cross section about the longitudinal tow axis. Systematic variations of each tow genus are calculated by a process known as reference period collation. In this process, each tow in the experimental specimen is collected according to genus type. Suitable vector translations are then performed to transform the tows into approximately the same location and phase. Systematic variations for each tow genus are represented by the collection of averages of the preceding tow variables at each position along the tow axis. Stochastic variations can be described by the root mean squared deviation from the systematic mean and the correlation length (the maximum distance at which a tow is still influenced by behavior further down the tow). ${ }^{1}$ The resulting statistical variables are then used as input to a geometry generator to construct a series of points defining the tow center and the corresponding surface profile. The model employed in the present study was restricted to having only systematic deviations.

Using the three-dimensional surface profile for each fiber tow (consisting of triangular facets joined together to form a continuous surface), a solid three-dimensional mesh comprising four-noded tetrahedral elements was generated using TetGen (Version 1.4.3). ${ }^{8}$ This meshing algorithm allows specification of constraints on element volume and aspect ratio, thereby facilitating construction of a range of numerically feasible meshes with varying degrees of mesh refinement. The output from TetGen is an array of $x y z$ nodal points and a list of element nodes describing tetrahedrons for each tow. Each tow is meshed individually and can be collated into a single model mesh for use in FE simulations. Tow interactions are governed by prescribed contact conditions, as described below. Comparisons of surface topographies obtained from this generation procedure and measured via DIC are shown in Fig. 7. The agreement is excellent over most of the area. The slight discrepancies are due in part to the elliptical idealization of the tow cross section in the translation of the CT images into the virtual sample. ${ }^{1}$ Some error also derives from the inability of DIC to correlate near surface discontinuities (e.g., edges of tows).

Finite element simulations were conducted in ABAQUS Standard (Version 6.9-EF1; Dassault Systèmes, Vélizy-Villacoublay, France). Following a successful mesh convergence study, a mesh comprising $2.2 \times 10^{6}$ first-order tetrahedral elements (C3D4) was used. For each element, a local coordinate system was defined so that the 1-direction was aligned with the local trajectory of the fiber tow. To prevent rigid body translation and rotation of the entire specimen, select translational degrees of freedom for three nodes within the mesh (each belonging to a different tow) were constrained. Surface-to-surface interactions were specified between neighboring tows to capture the effect of the SiC matrix (see Section III(3) for further detail). Surfaces were not adjusted ${ }^{\dagger}$ prior to application of the temperature change $(\Delta T=1000 \mathrm{~K}$, to match the experimental temperature profile). The effects of a through-thickness temperature gradient of $90 \mathrm{~K}$ were also simulated. These yielded surface strains that differed from those for isothermal simulations by $<1 \%$.

\section{(2) Constitutive Behavior}

The strains are assumed to be elastic and, because of symmetry, the tows are treated as being transversely isotropic. For consistency with previous descriptions of the microstructure, ${ }^{1}$

†A commonly employed feature in ABAQUS is the "strain-free adjustment" for surfaces that are to be tied together; nodes/elements on adjacent surfaces that are roughly, but not exactly, coincident are brought into closer contact without imposing any strain on the elements. 


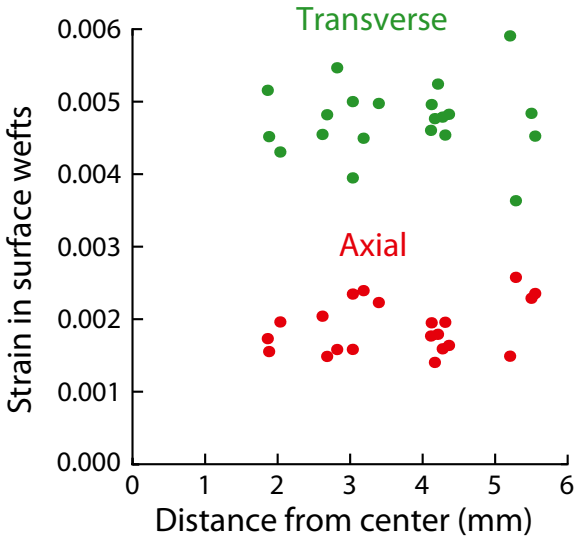

(a)

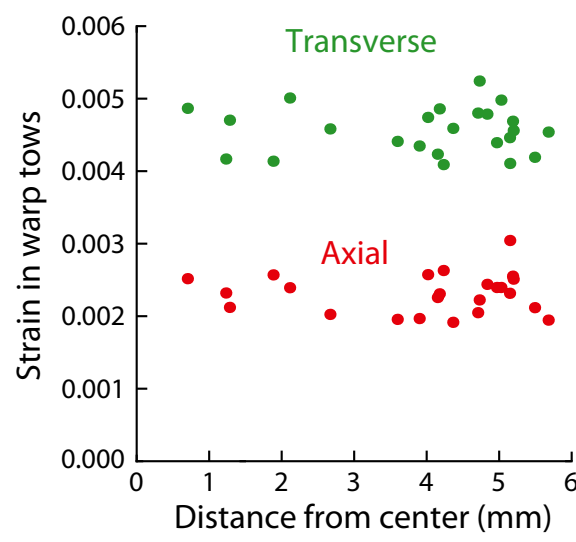

(b)

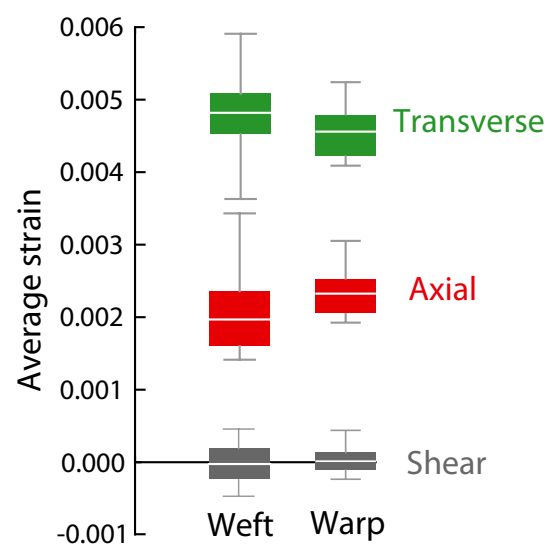

(c)

Fig. 6. Effects of measurement location on strains in (a) surface and (b) warp tows. (c) Box-and-whisker plot summarizing all distributions.

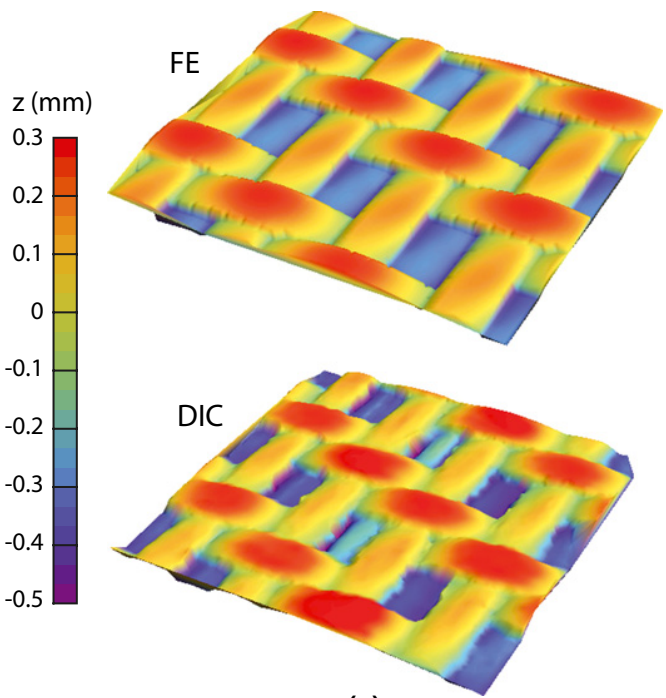

(a)

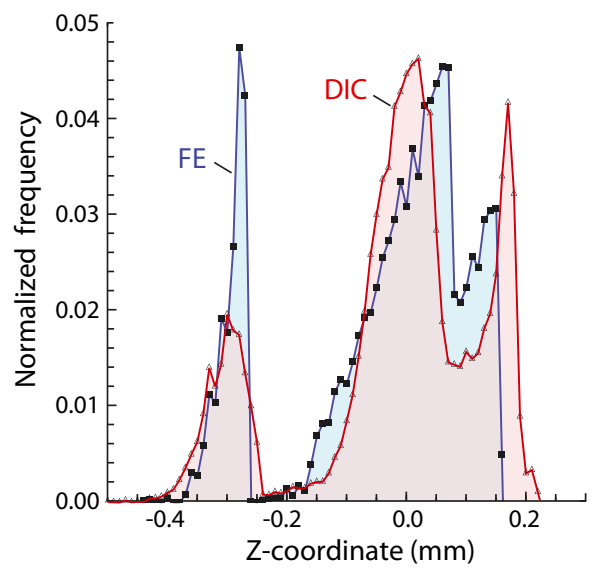

(b)

Fig. 7. (a) Comparison of surface topography obtained from the DIC measurements and the virtual geometry generator. (b) Quantitative assessment of the resulting surface height distributions.

the tow surface is taken to be the interior surface of the $\mathrm{SiC}$ jacket. The jacket material and the $\mathrm{SiC}$ within the tow interior are homogenized and represented by an effective medium that yields the same tow response. As the model only captures the architecture of the composite at the tow-scale, finescale microstructural features within the tows are ignored. The elastic constants of the tows were estimated using a combination of micromechanical models, ${ }^{9,10}$ mechanical tests, and FE simulations. The models are based on the assumption that each tow comprises unidirectionally aligned fibers in an effective medium that embodies the properties of the $\mathrm{SiC}$ and the intratow porosity. The effective medium properties were determined through comparisons of virtual tests of uniaxial tensile loading with corresponding experimental measurements. The models and calibration procedures are detailed in the Appendix. The resulting elastic constants are summarized in Table I.

The thermal expansion coefficients of the tows were determined using analogous micromechanical models, ${ }^{10}$ also detailed in the Appendix. The models take as inputs the CTEs and elastic properties of the composite constituents, where the constituents are now the fibers and the effective medium determined from the mechanical analysis. Two cases were considered, distinguished by the fiber axial CTE. In the first, the axial CTE was taken to be $-0.6 \times 10^{-6} \mathrm{~K}^{-1}$ (reported by the manufacturer, for near-ambient temperatures).
Table I. Elastic Properties of Tows

\begin{tabular}{lccccc}
\hline$E_{x}$ & $E_{y}$ & $G_{x y}$ & $G_{y z}$ & $v_{x y}$ & $v_{y z}$ \\
\hline $179 \mathrm{GPa}$ & $29 \mathrm{GPa}$ & $29 \mathrm{GPa}$ & $10 \mathrm{GPa}$ & 0.23 & 0.47 \\
\hline
\end{tabular}

This yields tow CTE values of $\alpha_{1}=0.5 \times 10^{-6} \mathrm{~K}^{-1}$ and $\alpha_{2}=6.5 \times 10^{-6} \mathrm{~K}^{-1}$. Other studies have shown a slight temperature sensitivity, with values of fiber axial CTE of about zero. ${ }^{11}$ Using this value, the composite CTEs are $\alpha_{1}=1.0 \times 10^{-6} \mathrm{~K}^{-1}$ and $\alpha_{2}=6.5 \times 10^{-6} \mathrm{~K}^{-1}$. As noted in forthcoming discussion, virtual tests based on these two sets of CTE values yield transverse strains that agree well with the measurements when the tow contact interaction is selected accordingly (as described in the following section); but the axial strains remain low compared to experimental measurements.

Motivated by this discrepancy, an alternative selection was made for the tow axial CTE. Here, the variations in the predicted axial strains with the axial tow CTE $\left(0.5 \times 10^{-6}\right.$ and $1.0 \times 10^{-6} \mathrm{~K}^{-1}$ ) were extrapolated linearly to the point where the predictions coincided with the measurements for the axial strains of the warp population of tow segments. The resulting inferred axial tow CTE is $\alpha_{1}=1.6 \times 10^{-6} \mathrm{~K}^{-1}$. Although the absolute value of this CTE remains small 
relative to those of most ceramics (including $\mathrm{SiC}$ ), it exceeds the micromechanically based predictions by a measurable amount $\left[(0.6-1.1) \times 10^{-6} \mathrm{~K}^{-1}\right]$.

\section{(3) Tow Contacts and Interactions}

As evident in the micrographs in Figs. 1 and 2, the individual tows are connected to their neighbors only in select regions. For instance, in Fig. 1(b), the surface wefts situated directly across from the crowns of the warp tows are not connected to the other tows in the plane of this section. The remaining wefts exhibit some contact with both the warp tows and the neighboring wefts. In another case [Fig. 1(d)], in a section taken along the weft direction through the center of a surface weft, the warp tows are in intimate contact with the weft tows. In contrast, in parallel sections away from the weft mid-line [Fig. 1(c)], the outermost warp tows make minimal contact with the wefts. These contacts were treated in the FE simulations using two approaches, intended to bind the realistic range of expected behavior.

In the first, the contacting regions between warp and weft tows were treated as being unbonded and constrained from moving only by friction: a limiting condition that would be obtained if all interfaces between tows were cracked (as a result of thermal stresses, for example). The friction coefficients were taken as either 0 or 0.5 (the choice proved unimportant, however, as demonstrated in a subsequent section). In the second, the tie interaction property in Abaqus was used between the nodes on the surfaces of the warp and weft tows, simulating a perfectly bonded interface. This interaction property takes as an argument a threshold nodal separation distance, hereafter refered to as the tie tolerance, below which any pair of nodes is considered to be rigidly attached throughout the simulation. Tie tolerances were varied over the range $0.5-75 \mu \mathrm{m}$. By comparison, the physical characteristic that most closely relates to the tie tolerance-notably, the thickness of the $\mathrm{SiC}$ coating on the tows - is about 30 $40 \mu \mathrm{m}$. If the CVI process produced a uniform SiC coating of this thickness, tows within $60-80 \mu \mathrm{m}$ of one another would be expected to be bonded. Thus, $75 \mu \mathrm{m}$ represents a physically realistic upper limit on the tie tolerance. In all cases, the nodes between adjacent warp tows were tied to one another where they were in contact with one another.

The effects of tie tolerance on the contact patches formed between adjacent tows are illustrated in Fig. 8 for the three representive tow types. Each of the tows has been bisected along its longitudinal axis and the two halves separated to reveal the contacts on both top and bottom surfaces. The contact patches are color-coded, as indicated by the legend. For a tie tolerance of $0.5 \mu \mathrm{m}$, the contact patches (in red) are typically about half a tow width. For the central weft, the locations of the patches made by contact with the warp tows alternate between the fore and the aft regions of the tow. A similar pattern is evident on the bottom side of the surface weft. However, on its top side, the contacts are made in the central region of the tow. For the warp tows, the contact patches made with the wefts are somewhat more irregular in size and shape, reflecting variations in trajectories of the weft tows. As the tie tolerance is increased to $5 \mu \mathrm{m}$, the contact patches expand only very slightly, now encompassing the regions shown in both red and green. The areas grow progressively but nonlinearly with tie tolerance. The degree of contact increases by a factor of about two as the tie tolerance is varied over the full range. Its effect is manifested in the thermal strains predicted by the virtual tests, presented in the following section.

\section{Virtual Tests Versus Experimental Measurements}

The first set of virtual tests was designed to probe the effects of tie tolerance on thermal strains in the warp and the surface weft segments, all for $\alpha_{1}=0.5 \times 10^{-6} \mathrm{~K}^{-1}$ and $\alpha_{2}=6.5 \times 10^{-6} \mathrm{~K}^{-1}$ (as determined from the micromechanical models and the initial estimates of tow properties). These results and the experimental measurements are plotted in Figs. 9(a) and (b). When the tows are allowed to slide (either without friction or with a friction coefficient of 0.5 ), the transverse tow strains are grossly over-estimated and the axial strains are grossly underestimated. Furthermore, in contrast to the experiments, the virtual tests predict virtually no strain variations. Consequently, this set of tow property values and tie conditions were deemed inadequate. The correlations improve as the contacts are tied and as the tie tolerance is increased. When the most realistic estimate of tie tolerance $(75 \mu \mathrm{m})$ is used, the computed transverse strains are comparable to the experimental measurements. Furthermore, the computed variations in transverse strains appear to be remarkably similar to those obtained experimentally. However, the computed axial strains remain in rather poor agreement with the experimental measurements.

The results of the virtual tests based on the tow axial CTE obtained by extrapolation are plotted in Figs. 9(c) and (d). Here, the computed axial strains for both warp and weft tows appear to be in good agreement with the measurements. The computed strain variations also appear to be of the correct magnitude (though slightly smaller than those obtained in the experiments, the difference being approximately equal to the magnitude of the strain measurement error of $\pm 0.0001-0.0003)$. In addition, the computed transverse tow strains are only very slightly affected, and hence, remain in agreement with the measurements. The inferred axial tow CTE is only very slightly higher than that obtained from micromechanical models and best estimates of the constituent properties $\left(1.6 \times 10^{-6} \mathrm{~K}^{-1}\right.$ vs $\left.1.0 \times 10^{-6} \mathrm{~K}^{-1}\right)$.

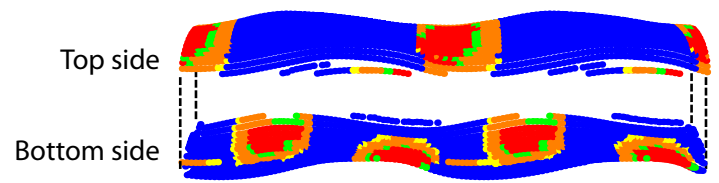

(a) Surface weft

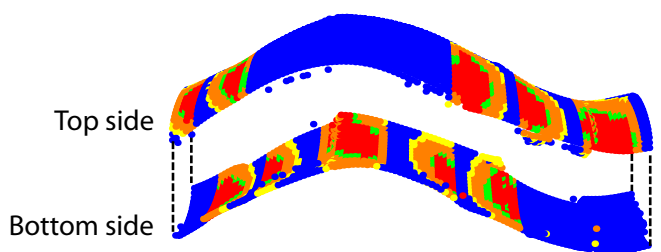

(c) Warp tow

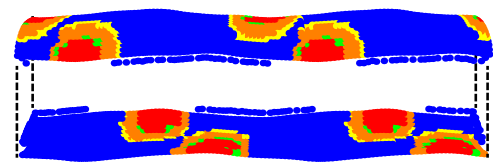

(b) Central weft

Fig. 8. Effects of tie tolerance on regions of tow contact. 

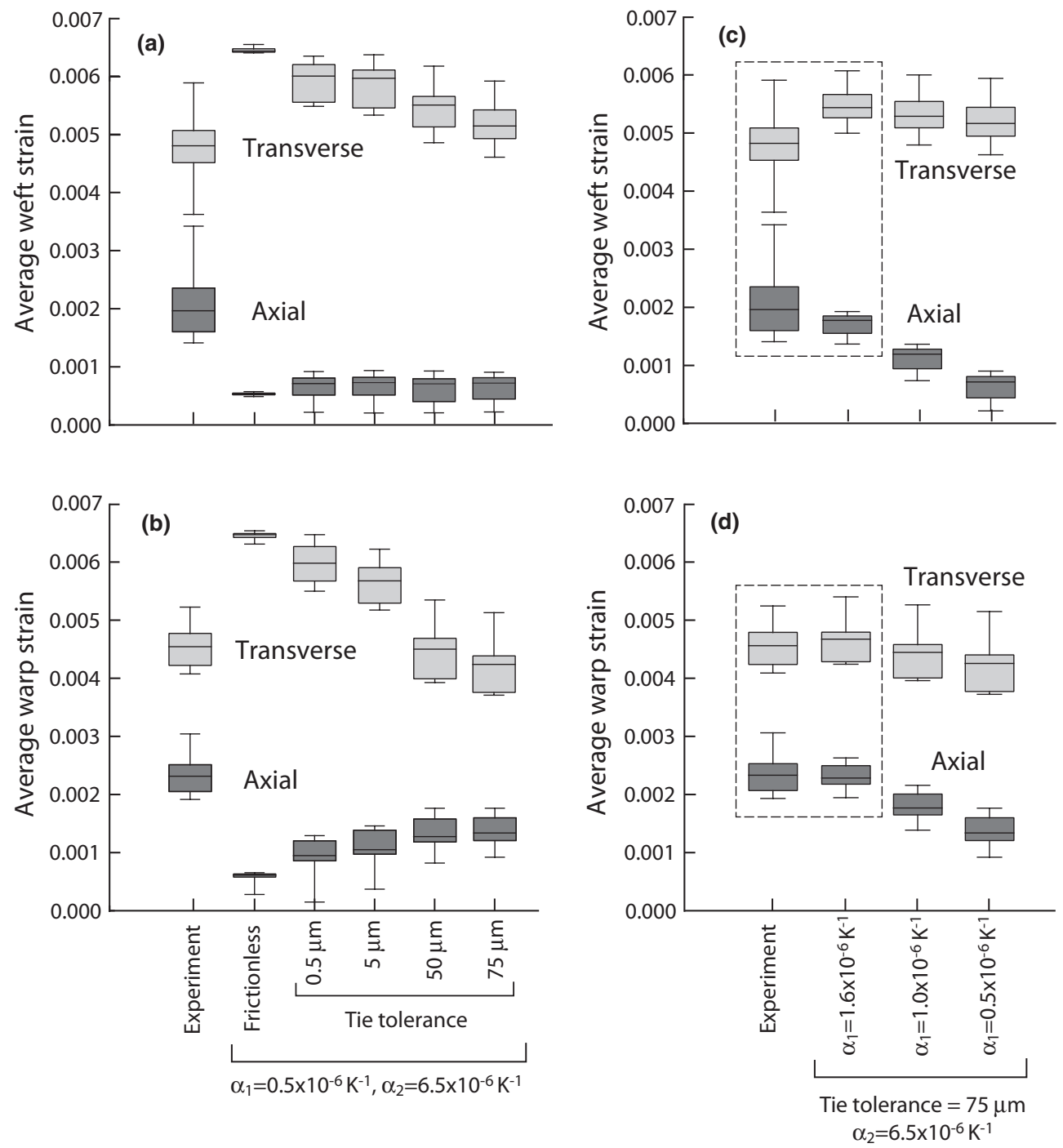

Fig. 9. Effects of (a) and (b) tie tolerance and (c) and (d) tow CTE values on computed tow segment strains (the results for frictional sliding with friction coefficient of 0.5 are identical to those for the frictionless case and thus have not been plotted here). The best correlations between experiments and simulations are contained within the dashed boxes.

Despite the seemingly small amount of $\mathrm{SiC}$ in the present $\mathrm{C} / \mathrm{SiC}$ composite, its effect on thermal strains is significant. The values expected of the fabric alone would be virtually identical to those of the individual fibers in the axial and radial directions: in the range of -0.0006 to 0 in the axial direction and about 0.009 in the transverse direction. The differences between these strains and those measured on the composite indicate a significant role of the $\mathrm{SiC}$ matrix.

\section{Conclusions}

A methodology for calibrating the thermoelastic properties of constituent tows in an anisotropic $\mathrm{C} / \mathrm{SiC}$ composite based on a combination of real and virtual tests has been presented. Because of the non-uniformity of the matrix phase and the presence of micro-cracks, tow properties cannot be reliably predicted a priori using micromechanical models alone; instead, some can only be inferred from coupled experimental/numerical studies of the kind presented here. A small number of iterations of FE simulations is required to achieve satisfactory agreement in all thermal strain components. The number of iterations is minimized by first performing simulations using model-based estimates of the effective tow properties, followed by judicious adjustments to select property values.
The study reveals that although each segment of a particular tow genus is nominally equivalent to all others, the strains within each group exhibit considerable variations. This is attributable to variations in both tow geometry and connectivity between adjacent tows. The virtual tests predict strain variations of comparable magnitude.

Although the present study has focused on a partially processed composite, the expectation is that the resulting thermoelastic constants will be relevant to composites with more heavily densified matrices. That is, since the tow interiors have been essentially sealed by the CVI SiC jacket, subsequent processing steps, involving slurry and precursor infiltration, will generate additional matrix material only in the large intertow spaces, leaving the intratow spaces unchanged. The major change to be implemented in the corresponding virtual tests is the addition of a matrix phase between the tows.

\section{Appendix \\ Thermoelastic Properties of Tows}

\section{A1. Elastic Constants}

The elastic properties of the $\mathrm{C} / \mathrm{SiC}$ tows are obtained in the following way. The $\mathrm{SiC}$ and the intra-tow porosity are 
Table A1. Elastic Properties of T300-Carbon Fibers

\begin{tabular}{|c|c|c|c|c|c|c|c|}
\hline \multicolumn{2}{|c|}{ Young's modulus ${ }^{\dagger}$} & \multicolumn{2}{|c|}{ Shear modulus ${ }^{\dagger}$} & $\frac{\text { Bulk modulus }^{\ddagger}}{k_{f \perp}}$ & \multicolumn{2}{|c|}{ Poisson's ratio } & $\frac{\text { Volume fraction }}{f^{\pi}}$ \\
\hline $230 \mathrm{GPa}$ & $14.5 \mathrm{GPa}$ & $22.75 \mathrm{GPa}$ & $4.8 \mathrm{GPa}$ & $15 \mathrm{GPa}$ & 0.27 & 0.51 & 0.61 \\
\hline
\end{tabular}

${ }^{\dagger}$ BP-Amoco chemicals.

${ }^{\ddagger} E_{f \perp}=\frac{4 k_{f \perp} G_{f \perp}}{k_{f \perp}+m G_{f \perp}}, m=1+\frac{4 k_{f \perp} v_{f \|}^{2}}{E_{f l}}$.

${ }^{s_{f \perp}} v_{f}=\left(\mathrm{E}_{f \perp} \perp / 2 G_{f \perp}\right)-1$.

"Determined from filament count and fiber density reported by the manufacturer in combination with the tow cross-sectional area measured by computed tomography. ${ }^{1}$

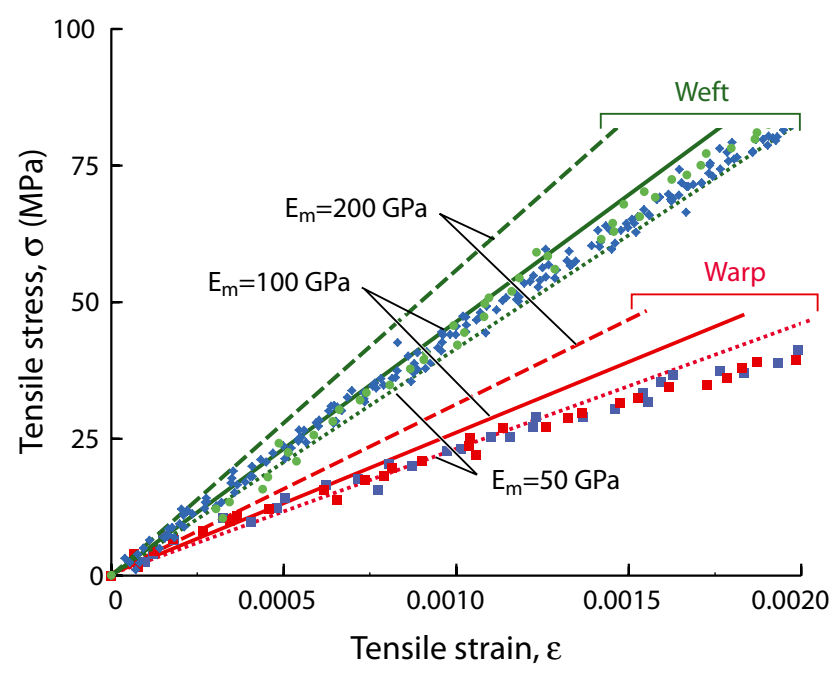

Fig. A1. Calibration of effective medium Young's modulus for warp and weft loading. The solid symbols are experimental data points and the lines are results of finite element calculations. Only the small strain parts of the full stress-strain curves are shown.

homogenized into an effective medium and the properties of this medium are inferred from experimental measurements and FE simulations of tensile properties in both warp and weft orientations. The calibration procedure is performed in three steps: (i) the tow properties are calculated using established composite models, the reported fiber properties (Table A1) and a set of assumed elastic properties of the effective medium; (ii) FE calculations are performed of the elastic response of the composite in both orientations using the calculated tow properties; and (iii) the FE results are compared with the experimental measurements. The procedure is repeated until consistency is obtained between the computed and measured composite moduli.

The tows are treated as being transversely isotropic. Denoting $x$ as the fiber direction and $y$ and $z$ as the two orthogonal directions, the pertinent elastic constants are the Young's moduli, $E_{x}$ and $E_{y}$, the shear moduli, $G_{x y}$ and $G_{y z}$, the bulk modulus, $k$, and the Poisson's ratios, $v_{x y}$ and $v_{y z}$. Among these, five are necessary for a complete description of the tow response. The constants are calculated from Hashin's equations for composites reinforced with unidirectionally aligned anisotropic fibers. ${ }^{10}$

Closed-form solutions for the elastic constants $E_{x}, G_{x y}, v_{x y}$ and $k$ for composites with transversely isotropic fibers are based on modifications to the solutions from the composite cylinder assemblage model for isotropic constituents. ${ }^{9,10}$ They are given by:

$$
E_{x}=E_{f||} f+E_{m}(1-f)+\frac{4\left(v_{f \|}-v_{m}\right)^{2} f(1-f)}{\frac{(1-f)}{k_{f \perp}}+\frac{f}{k_{m}}+\frac{1}{G_{m}}}
$$

$$
\begin{aligned}
& v_{x y}=v_{f||} f+v_{m}(1-f)+\frac{\left(v_{f \|}-v_{m}\right)\left(\frac{1}{k_{m}}+\frac{1}{k_{f \perp}}\right) f(1-f)}{\frac{(1-f)}{k_{f \perp}}+\frac{f}{k_{m}}+\frac{1}{G_{m}}} \\
& G_{x y}=G_{m}+\frac{f}{\frac{1}{G_{f \mid}-G_{m}}+\frac{1-f}{2 G_{m}}} \\
& k=k_{m}+\frac{f}{\frac{1}{k_{f \perp}-k_{m}}+\frac{1-f}{k_{m}+G_{m}}}
\end{aligned}
$$

where the subscripts $\|$ and $\perp$ denote axial and transverse directions, respectively, $f$ is the fiber volume fraction and the subscript $m$ denotes the effective medium.

Although closed-form solutions for the remaining parameters $-G_{y z}, E_{y}$ and $v_{y z}$ - do not exist, the available bounds are close to one another and provide a useful basis for estimation. Here, we employ average values of the upper and lower bounds. Provided $G_{f} \perp<G_{m}$ and $k_{f}<k_{m}$ (as in the system of present interest), the bounds for the transverse shear modulus, $G_{y z( \pm)}$ are given by:

$$
\begin{aligned}
& G_{y z(-)}=G_{m}\left[1+\frac{\left(1+\beta_{1}\right) f}{\rho-f\left(1+\frac{3 \beta_{1}^{2}(1-f)^{2}}{\alpha f^{3}-\beta_{1}}\right)}\right] \\
& G_{y z(+)}=G_{m}+\frac{f}{\frac{1}{G_{f \perp}-G_{m}}+\frac{k_{m}+2 G_{m}}{2 G_{m}\left(k_{m}+G_{m}\right)}(1-f)}
\end{aligned}
$$

where \pm represents the upper or lower bound and $\alpha=\frac{\beta_{1}-\gamma \beta_{2}}{1+\gamma \beta_{2}}$; $\rho=\frac{\gamma+\beta_{1}}{\gamma-1} ; \quad \beta_{1}=\frac{k_{m}}{k_{m}+2 G_{m}} ; \quad \beta_{2}=\frac{k_{f}}{k_{f}+2 G_{f \perp}} ; \gamma=\frac{G_{f \perp}}{G_{m}}$. The bounds for $E_{y}$ and $v_{y z}$ are:

$$
\begin{aligned}
& E_{y( \pm)}=\frac{4 k G_{y z( \pm)}}{k+m G_{y z( \pm)}} \\
& v_{y( \pm)}=\frac{k-m G_{y z(\mp)}}{k+m G_{y z(\mp)}}
\end{aligned}
$$

where $m=1+\frac{4 k v_{x y}}{E_{x}}$.

The Poisson's ratio of the effective medium is taken to be $v_{m}=0.2$ and its Young's modulus $E_{m}$ is varied over a wide range. For each selected value of $E_{m}$, the effective tow prop-

Table A2. Thermal Expansion Coefficients of Composite Constituents

\begin{tabular}{lc}
\hline$\alpha_{m}{ }^{13}$ & $\alpha_{f \|}^{\dagger}{ }^{\dagger}$ \\
\hline $4.3 \times 10^{-6} \mathrm{~K}^{-1}-0.6 \times 10^{-6} \mathrm{~K}^{-1}$ & $9 \times 10^{-6} \mathrm{~K}^{-1}$
\end{tabular}


erties are computed from Eqs. (A1)-(A8) and used as inputs to the finite element calculations.

Comparisons of the experimental measurements and the numerical results are plotted in Fig. A1. Focusing on the very small strain regime, the comparisons indicate that the best estimate of the effective medium modulus is $E_{m} \approx 100 \mathrm{GPa}$ (it should be noted, however, that the composite modulus is only weakly dependent on this selection and thus the inferred estimate is subject to considerable uncertainty). The resulting average tow properties are summarized in Table I in the main body of the article.

\section{A2. Thermal Expansion Coefficients}

The thermoelastic properties of the tows are estimated from micromechanical models using the coefficients of thermal expansion of the constituents, found in Table A2, and the tow mechanical properties calculated in Appendix A1. The thermal expansion coefficient of the effective medium is assumed to be the same as that of CVI SiC. A comprehensive presentation can be found elsewhere. ${ }^{10,12}$ For composites with transversely isotropic fibers, the axial and transverse coefficients of thermal expansion, $\alpha_{x}$ and $\alpha_{y}$, are given by:

$$
\begin{aligned}
& \alpha_{x}=\bar{\alpha}_{\|}+\left(\alpha_{k l}^{f}-\alpha_{k l}^{m}\right) P_{k l r s}\left(S_{r s 11}^{c}-\bar{S}_{r s 11}\right) \\
& \alpha_{y}=\bar{\alpha}_{\perp}+\left(\alpha_{k l}^{f}-\alpha_{k l}^{m}\right) P_{k l r s}\left(S_{r s 22}^{c}-\bar{S}_{r s 22}\right)
\end{aligned}
$$

where $S$ denotes the elastic compliance tensor (computed using the results in Appendix A1) and the superscripts $f, m$, and $c$ refer to fiber, effective medium and composite, respectively. The overbar denotes phase average, notably:

$$
\begin{aligned}
& \bar{\alpha}_{\|}=\alpha_{\| f}^{f} f+\alpha_{\|}^{m}(1-f) \\
& \bar{\alpha}_{\perp}=\alpha_{\perp}^{f} f+\alpha_{\perp}^{m}(1-f) \\
& \bar{S}_{r s i j}=S_{r s i j}^{f} f+S_{r s i j}^{m}(1-f)
\end{aligned}
$$

and the tensor $P$ is the inverse of the difference between fiber and effective medium compliance tensors:

$$
P_{k l r s}\left(S_{r s i j}^{f}-S_{r s i j}^{m}\right)=I_{k l i j}
$$

where $I$ is the identity tensor. ${ }^{10}$

\section{Acknowledgments}

This work was supported by the US AFOSR (Ali Sayir) and NASA (Anthony Calomino) under the National Hypersonics Science Center for Materials and Structures (AFOSR Prime contract no. FA9550-09-1-0477 to Teledyne Scientific and Sub-contract no. B9U538772 to UCSB). We gratefully acknowledge David Marshall (Teledyne Scientific) for providing the materials used in this study, for numerous fruitful discussions and for critical comments on the manuscript. We also acknowledge support from the Center for Scientific Computing at the CNSI and MRL: an NSF MRSEC (DMR-1121053) and NSF CNS-0960316.

\section{References}

${ }^{1}$ H. Bale, M. Blacklock, M. R. Begley, D. B. Marshall, B. N. Cox, and R. O. Ritchie, "Characterizing Three-Dimensional Textile Ceramic Composites Using Synchrotron X-Ray Micro-Computed-Tomography," J. Am. Ceram. Soc., 95 [1] 392-402 (2012).

${ }^{2}$ M. Blacklock, H. Bale, M. Begley, and B. Cox, "Generating Virtual Textile Composite Specimens Using Statistical Data from Micro-Computed Tomography: 1D Tow Representations for the Binary Model," J. Mech. Phys. Solids, 60 [3] 451-70 (2012).

${ }^{3}$ R. G. Rinaldi, M. Blacklock, H. Bale, M. R. Begley, and B. N. Cox, "Generating Virtual Textile Composite Specimens Using Statistical Data from Micro-Computed Tomography: 3D Tow Representations," J. Mech. Phys. Solids, 60 [8] 1561-81 (2012).

${ }^{4}$ M. Blacklock, R. G. Rinaldi, and B. Cox, Virtual Specimen Generator. 2013a. Available at: http://www.engineering.ucsb.edu/ mblacklock/VSG/ (accessed August 2012).

${ }^{5}$ M. D. Novak and F. W. Zok, "High-Temperature Materials Testing with Full-Field Strain Measurement: Experimental Design and Practice," Rev. Sci. Instrum., 82 [11] 115101, 6pp (2011).

${ }^{6}$ V. P. Rajan, M. N. Rossol, and F. W. Zok, "Optimization of Digital Image Correlation for High-Resolution Strain Mapping of Ceramic Composites," Exp. Mech., 52 [9] 1407-21 (2012).

${ }^{7}$ J. J. Orteu, T. Cutard, D. Garcia, E. Cailleux, and L. Robert, "Application of Stereovision to the Mechanical Characterisation of Ceramic Refractories Reinforced with Metallic Fibres," Strain, 43 [2] 96-108 (2007).

${ }^{8}$ S. Hang, TetGen 1.4.3. Available at: tetgen.org (accessed April 2013)

${ }^{9} \mathrm{Z}$. Hashin and B. W. Rosen, "The Elastic Moduli of Fiber-Reinforced Materials," J. Appl. Mech., 31 [12] 223-32 (1964).

${ }^{10} \mathrm{Z}$. Hashin, "Analysis of Properties of Fiber Composites with Anisotropic Constituents," J. Appl. Mech., 46 [3] 543-50 (1979).

${ }^{11}$ M. Trinquecoste, J. L. Carlier, A. Derre, P. Delhaes, and P. Chadeyron, "High Temperature Thermal and Mechanical Properties of High Tensile Carbon Single Filaments," Carbon, 34 [7] 923-9 (1996).

${ }^{12} \mathrm{~V}$. M. Levin, "On the Coefficients of Thermal Expansion of Heterogeneous Materials," Mech. Solids, 2 [1] 58-61 (1967).

${ }^{13}$ E. Lara-Curzio, S. S. Sternstein, C. R. Hubbard, B. Cavin, and W. Porter, "High-Temperature Structural Stability of Chemically Vapor-Deposited Silicon-Carbide Filaments," Mater. Sci. Eng., A, 172 [1-2] 167-71 (1993). 